\title{
Analysis of the association of diabetes mellitus with cancer using autopsy records
}

\author{
RYUICHI WADA ${ }^{1,2}$, SOTAI KIMURA ${ }^{3}$, SHOKO KURE $^{1,2}$, KOUSUKE ISHINO $^{1}$, \\ MITSUHIRO KUDO $^{1}$ and ZENYA NAITO ${ }^{1,2}$ \\ ${ }^{1}$ Department of Integrated Diagnostic Pathology, Nippon Medical School; \\ ${ }^{2}$ Department of Diagnostic Pathology, Nippon Medical School Hospital, Tokyo 113-8602; \\ ${ }^{3}$ Faculty of Medicine, Hirosaki University School of Medicine, Hirosaki 036-8601, Japan
}

Received April 8, 2020; Accepted May 11, 2020

DOI:10.3892/wasj.2020.52

\begin{abstract}
In the present study, the association of diabetes mellitus (DM) with cancer was investigated using autopsy records. Among the 83,829 autopsy records between the years 2005 and 2009, 74,869 cases $\geq 40$ years were eligible for the analysis. The cases with DM and cancers were selected, and the risk of cancer was estimated as the odds ratio (OR) by logistic regression analysis with the adjustment of age. Among the 74,869 cases, there were 49,676 males and 25,193 females. DM was noted in 4,901 males (9.9\%) and 2,066 females $(8.2 \%)$. In the liver, the OR for hepatocellular carcinoma (HCC) was increased to 1.25 (95\% confidence interval (CI), $1.12-1.39, \mathrm{P}<0.001)$ and to $1.22(95 \% \mathrm{CI}, 1.00-1.49, \mathrm{P}<0.05)$ in males and females with DM, respectively. The ORs for invasive ductal carcinoma of the pancreas, cancers of the thyroid and kidney were not increased, and the ORs for cancers of the lung, stomach, colon/rectum, biliary tract, prostate, breast and uterus appeared to be decreased. The analyzed autopsy cases corresponded to only $1.4 \%$ of deceased individuals aged $\geq 40$ years, and the ORs for cancers in DM estimated using the autopsy records may warrant a careful consideration. To the best of our knowledge, the present study is the first to confirm the increased OR for HCC in DM by a cross-sectional analysis using autopsy records.
\end{abstract}

\section{Introduction}

Diabetes mellitus (DM) is a risk factor for the development of cancer $(1,2)$. There may be a difference in the increase of the risk of cancer among the organs in DM, and the increased risk of developing cancer of the liver and pancreas has been noted

Correspondence to: Dr Ryuichi Wada, Department of Integrated Diagnostic Pathology, Nippon Medical School, 1-1-5 Sendagi, Bunkyo-ku, Tokyo 113-8602, Japan

E-mail:w-ryuichi@nms.ac.jp

Key words: odds ratio, epidemiology, prevalence, hepatocellular carcinoma, intrahepatic cholangiocarcinoma in both males and females with DM $(3,4)$. The association of DM with cancer has been investigated in cohort studies using large-scale populations and in case-control study (5-8). The association of DM with cancer has not yet been examined using autopsy records.

Autopsy cases in Japan have been recorded and published as the Annual of the Pathological Autopsy Cases in Japan by Japanese Society of Pathology since 1960 (9). Each autopsy record contains clinical and pathological diagnoses. Pathological diagnosis includes the diagnosis that developed during the lifetime of the patient. Even in cases in which DM was not clinically evident during the patient's lifetime, a diagnosis of DM is made at the time of autopsy, depending on the pathological findings. The pathological diagnosis contains the present and past history of cancers and includes detailed histological data on cancer and latent cancer found at the autopsy.

In the present study, the association of DM with cancers was investigated using the autopsy records between 2005 and 2009 in Japan. The odds ratios (ORs) for cancers in DM cases were estimated. Although the study may warrant careful interpretation, the increase in the ORs of hepatocellular carcinoma (HCC) was verified using the autopsy records. To the best of our knowledge, the present study is the first to elucidate the association of DM with cancers using autopsy records.

\section{Materials and methods}

Autopsy records and selection of cases. The Annual of Pathological Autopsy Cases in Japan has been published by the Japanese Society of Pathology. The portable document format (PDF) of the autopsy records published between 2005 and 2009 was used in the present study (9). The use of the PDF files was permitted by the Information Committee of the Japanese Society of Pathology. Autopsy records of the age, sex, clinical diagnosis, pathological diagnosis of one autopsy case were converted to a spreadsheet file. A total of 83,829 cases were recorded in the Annual of Pathological Autopsy Cases in Japan between 2005 and 2009. All records were reviewed by two authors (RW and SKi).

The following cases were excluded from the estimation of ORs: i) Cases that were not published and lacked a description of age, sex and diagnosis; ii) cases of hermaphrodites; iii) cases 
of regional dissection of the head, chest, or abdomen and postmortem biopsy; iv) fetuses, stillborn infants; and v) cases $<40$ years of age for the comparison with the previous cohort and case-control studies (5-8), in which ORs for cancers in DM were estimated in individuals $\geq 40$ years.

Identification of diabetes mellitus and cancer cases. The cases of DM and cancer were identified by a text search. DM cases were identified by the words 'diabetes', 'diabetes mellitus', 'DM', 'insulin dependent diabetes mellitus (IDDM)', 'non-insulin dependent diabetes mellitus (NIDDM)', 'type 1 diabetes', 'type 2 diabetes', 'Kimmelstiel-Wilson syndrome' and 'diabetic nephropathy' in clinical and pathological diagnoses. The cases without DM was designated as non-DM.

Cancers were identified by the combination of the words 'cancer' or 'carcinoma' and the words of the organs 'thyroid', 'lung', 'stomach', 'colon', 'rectum', 'breast', 'kidney', 'uterus', 'ovary' and 'prostate'. Histological subtypes were taken into consideration in the liver and pancreas. HCC was identified by the words 'hepatocellular carcinoma' and 'HCC'. Intrahepatic cholangiocarcinoma (ICC) was identified by the words 'intrahepatic cholangiocarcinoma', 'cholangiocellular carcinoma (CCC)', 'ICC' and 'CCC'. Invasive ductal carcinoma (IDC) of the pancreas was identified by the words 'pancreatic ductal carcinoma', 'pancreas cancer', 'pancreas adenocarcinoma', and by the combination of 'invasive ductal carcinoma' and 'ductal carcinoma' with 'pancreas'.

Statistical analysis. Identified cases were stratified into two strata of age: Between 40 and 69 years, and $\geq 70$ years. The distribution of age was analyzed using the Chi-square test. Age-adjusted ORs and 95\% confidence intervals (CIs) of cancers were estimated with the logistic regression method. Analyses were performed using RStudio (RStudio, Inc., https://www.rstudio.com). P-values $<0.05$ were considered to indicate statistically significant differences.

\section{Results}

Autopsy cases. Among the 83,829 cases, a total 8,960 cases were excluded. The excluded cases were as follows: Cases that were not published and lacked a description of age, sex and diagnosis (427 cases); cases of hermaphrodites (5 cases); cases of regional dissection of the head, chest, or abdomen and postmortem biopsy (1,314 cases); fetuses, stillborn infants (3,533 cases); and cases younger than 40 years of age (3,681 cases). Finally, 74,869 were eligible for the analysis (Fig. 1).

Based on the Vital Statistics of Japan Final Data, General Mortality (10), the total number of deaths was 5,560,852 between 2005 and 2009 in Japan. The number of deaths of those $\geq 40$ years was $5,425,379$. Thus, the cases eligible for the analysis corresponded to $1.4 \%$ of deceased individuals $\geq 40$ years.

Among the 74,869 records, 49,676 were males and 25,193 were females (Fig. 1). A total of 6,967 cases (9.3\%) were identified as DM. DM was noted in 4,901 men (9.9\%) and 2,066 women $(8.2 \%)$. The cases were stratified into two strata: Between 40 and 69 years, and $\geq 70$ years. The distribution of

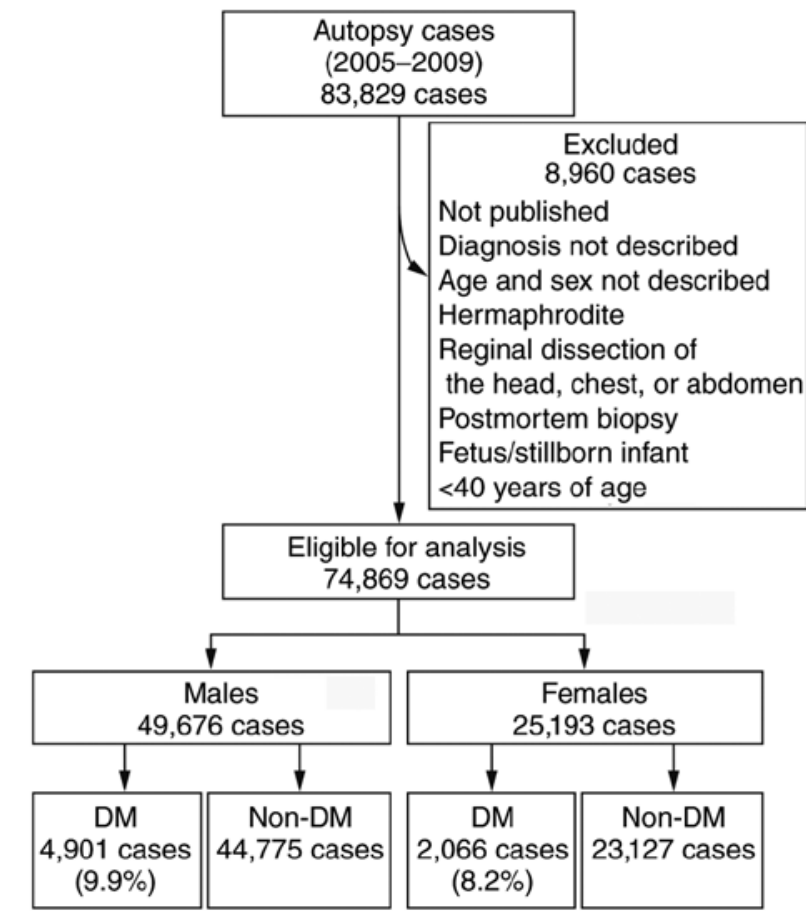

Figure 1. Flow chart showing the selection of autopsy cases. DM, diabetes mellitus.

the numbers of cases by age did not differ significantly between DM and non-DM in both males and females (Tables I and II).

Odds ratio for cancers in males. The ORs for cancer in males are presented in Table I. The age-adjusted ORs for cancers of the thyroid, colon/rectum and kidney were comparable. The ORs for cancers of the lung, stomach, biliary tract and prostate were significantly decreased in the DM cases. The OR for HCC was significantly increased $(1.25 ; 95 \% \mathrm{CI}, 1.12-1.39, \mathrm{P}<0.001)$ in DM, whereas the OR for ICC was decreased $(0.72 ; 95 \% \mathrm{CI}$, $0.52-1.00, \mathrm{P}<0.05)$. The OR for IDC of the pancreas was not considered increased $(1.04 ; 95 \% \mathrm{CI}, 0.89-1.21, \mathrm{P}=0.663)$. The OR for multiple cancers was decreased $(0.78 ; 95 \% \mathrm{CI}$, $0.70-0.88, \mathrm{P}<0.001)$ in males.

Odds ratio for cancers in females. The ORs for cancer in females are presented in Table II. The age-adjusted ORs for cancers of the thyroid and kidney were comparable. The ORs for cancers of the lung, stomach, colon/rectum, breast and uterus were decreased in DM cases. The OR for HCC in DM was significantly increased (1.22; 95\% CI: $1.00-1.49, \mathrm{P}<0.05)$. The OR for ICC was decreased $(0.44 ; 95 \%$ CI, 0.22-0.85, $\mathrm{P}<0.05)$. The OR for IDC of the pancreas was not considered increased $(1.03 ; 95 \% \mathrm{CI}, 0.83-1.29, \mathrm{P}=0.776)$. The OR for multiple cancers was comparable in women $(0.86$; $95 \% \mathrm{CI}$, 0.67-1.09, $\mathrm{P}=0.191$ ).

\section{Discussion}

The present study is the first, to the best of our knowledge, to investigate the association of DM with cancer using autopsy records. In the liver, the age-adjusted OR for $\mathrm{HCC}$ was significantly increased (1.25 and 1.22) in males and females with DM, 
Table I. Number of cancer cases and odds ratios in males.

\begin{tabular}{|c|c|c|c|c|}
\hline Cases & $\mathrm{DM}$ & Non-DM & Age-adjusted OR (95\% CI) & P-value \\
\hline Total no. of cases & 4,901 & 44,775 & & \\
\hline \multicolumn{5}{|l|}{ Age $(\text { years })^{\mathrm{a}}$} \\
\hline $40-69$ & 2,145 & 19,108 & & \\
\hline$\geq 70$ & 2,756 & 25,667 & & \\
\hline \multicolumn{5}{|l|}{ Cancers } \\
\hline Thyroid & 90 & 823 & $1.00(0.80-1.24)$ & 0.982 \\
\hline Lung & 465 & 6,749 & $0.59(0.54-0.65)$ & $<0.001$ \\
\hline Stomach & 336 & 4,226 & $0.71(0.63-0.80)$ & $<0.001$ \\
\hline Colon/rectum & 327 & 3,197 & $0.93(0.83-1.05)$ & 0.256 \\
\hline \multicolumn{5}{|l|}{ Liver } \\
\hline $\mathrm{HCC}$ & 414 & 3,076 & $1.25(1.12-1.39)$ & $<0.001$ \\
\hline ICC & 38 & 480 & $0.72(0.52-1.00)$ & 0.043 \\
\hline Pancreas IDC & 182 & 1,607 & $1.04(0.89-1.21)$ & 0.663 \\
\hline Biliary tract & 58 & 921 & $0.57(0.44-0.75)$ & $<0.001$ \\
\hline Kidney & 86 & 781 & $1.01(0.80-1.26)$ & 0.959 \\
\hline Prostate & 349 & 3,835 & $0.82(0.74-0.93)$ & $<0.001$ \\
\hline Multiple cancers & 328 & 3,776 & $0.78(0.70-0.88)$ & $<0.001$ \\
\hline
\end{tabular}

${ }^{\text {a }} \mathrm{P}=0.147$, data analyzed using the Chi square test. CI, confidence interval; DM, diabetes mellitus; HCC, hepatocellular carcinoma; ICC, intrahepatic cholangiocarcinoma; IDC, invasive ductal carcinoma; OR, odds ratio.

respectively. On the other hand, the OR for ICC was decreased (0.72 and 0.44) in males and females with DM, respectively. The difference in OR between histological subtypes of cancers in the liver was demonstrated. The ORs for cancers of other organs in DM appeared to be reduced in males and females. The use of autopsy records for the estimation of OR for cancers may warrant careful consideration.

In previous cohort studies in Japan, 'cancer' of the liver was identified by International Classification of Diseases (ICD) code C22 (Table III) (5-7). The ICD code C22 includes all histological subtypes of malignant tumor of the liver, the precise risk of the histological subtype was unclear. In a case-control study, the increase in OR for HCC was estimated from the cases with HCC (8). Although the ORs were lower than those in previous studies in Japan, the significant increase in OR for HCC in DM using autopsy records was in line with that of previous studies. The increase in OR for HCC was noted in both males and females in the present study. The association of DM with HCC was confirmed in the present study using autopsy records from the Annual of Pathological Autopsy Cases in Japan.

The ORs estimated in the present study appeared to be decreased. The increase in the risk of developing ICC in DM was suggested by a previous study (11). The increase in the risk of developing pancreatic cancer in DM was also previously suggested in cohort studies in Japan (5-7); however, the increase in OR for IDC of the pancreas was not noted in the present study. This may be in part due to the fact that the cases were selected by ICD code C25 in previous studies (5-7). Other neoplasms, such as intraductal papillary mucinous neoplasm and neuroendocrine neoplasms may be included in the code. If it is taken into consideration that the OR for $\mathrm{HCC}$ is also lower than those of the previous studies, the ORs estimated in the present study may be underestimated, compared with those in the previous studies, and the results warrant careful interpretation. In the autopsy records from the Annual of Pathological Autopsy Cases in Japan, the temporal sequence of diseases is not registered. It is not feasible to estimate the 'true' risk of cancer development, and the OR may need to be interpreted as the lifetime prevalence of cancer in DM cases. The ORs were adjusted only by age in the present study. The ORs were not adjusted with other important confounding factors, such as smoking, body weight and occupation, as these factors were not included in the records of the Annual of Pathological Autopsy Cases in Japan. These factors may need to be included in the Annual of Pathological Autopsy Records in Japan in the future. The analyzed autopsy cases correspond $1.4 \%$ of mortality between 2005 and 2009 . There is a bias for the selection of autopsy cases by attending doctors. These factors may affect the estimation of OR.

According to the National Health and Nutrition Survey in Japan 2007 (12), the prevalence of DM in Japanese individuals $\geq 40$ years was estimated to be $18.0 \%$ among males and $9.1 \%$ among females by the measurement of HbAlc. In the present study, the percentage of DM was estimated as $9.9 \%$ among males and $8.2 \%$ among females. The percentage of DM in the autopsy records was lower than that in the survey. However, the percentage was higher than those in previous cohort studies, in which DM was recorded in a self-report manner (Table III) (5-7). In some recent cases of DM, the pathological changes associated with DM may be subtle and overlooked. The pathological changes in DM were carefully evaluated by pathologists and registered in autopsy records. Although careful interpretation is required, autopsy records are valuable 
Table II. Number of cancer cases and ORs in females.

\begin{tabular}{|c|c|c|c|c|}
\hline Cases & $\mathrm{DM}$ & Non-DM & Age-adjusted OR (95\% CI) & $\mathrm{P}$-value \\
\hline Total no. of cases & 2,066 & 23,127 & & \\
\hline \multicolumn{5}{|l|}{ Age $(\text { years })^{\mathrm{a}}$} \\
\hline $40-69$ & 699 & 8,174 & & \\
\hline$\geq 70$ & 1,367 & 14,953 & & \\
\hline \multicolumn{5}{|l|}{ Cancers } \\
\hline Thyroid & 77 & 797 & $1.09(0.86-1.38)$ & 0.497 \\
\hline Lung & 104 & 1,672 & $0.68(0.55-0.83)$ & $<0.001$ \\
\hline Stomach & 62 & 1,283 & $0.52(0.40-0.68)$ & $<0.001$ \\
\hline Colon/rectum & 104 & 1,453 & $0.79(0.64-0.96)$ & 0.017 \\
\hline \multicolumn{5}{|l|}{ Liver } \\
\hline $\mathrm{HCC}$ & 117 & 1,077 & $1.22(1.00-1.49)$ & 0.045 \\
\hline $\mathrm{ICC}$ & 9 & 229 & $0.44(0.22-0.85)$ & 0.005 \\
\hline Pancreas IDC & 88 & 954 & $1.03(0.83-1.29)$ & 0.776 \\
\hline Biliary tract & 42 & 665 & $0.69(0.51-0.95)$ & 0.017 \\
\hline Kidney & 18 & 212 & $0.95(0.59-1.54)$ & 0.837 \\
\hline Breast & 63 & 945 & $0.74(0.57-0.96)$ & 0.020 \\
\hline Uterus & 25 & 437 & $0.64(0.43-0.96)$ & 0.023 \\
\hline Multiple cancers & 75 & 971 & $0.86(0.67-1.09)$ & 0.191 \\
\hline
\end{tabular}

${ }^{\mathrm{a}} \mathrm{P}=0.176$, data analyzed using the Chi square test. CI, confidence interval; DM, diabetes mellitus; HCC, hepatocellular carcinoma; ICC, intrahepatic cholangiocarcinoma; IDC, invasive ductal carcinoma; OR, odds ratio.

Table III. Odds ratios of cancers of the liver in DM.

\begin{tabular}{|c|c|c|c|c|c|c|c|c|}
\hline \multirow[b]{2}{*}{ Author } & \multirow{2}{*}{$\begin{array}{l}\text { No. of } \\
\text { cases }\end{array}$} & \multirow{2}{*}{$\begin{array}{c}\text { Age } \\
\text { (years) }\end{array}$} & \multirow{2}{*}{$\begin{array}{l}\text { Study } \\
\text { design }\end{array}$} & \multirow{2}{*}{$\begin{array}{c}\text { Case } \\
\text { selection }\end{array}$} & \multirow{2}{*}{$\begin{array}{l}\mathrm{DM} \\
(\%)\end{array}$} & \multicolumn{2}{|c|}{ OR $(95 \%$ CI $)$} & \multirow[b]{2}{*}{ (Refs.) } \\
\hline & & & & & & Males & Females & \\
\hline Inoue et al & 97,771 & $40-69$ & Cohort & ICD C22 & 6.7 & $\begin{array}{c}2.37 \\
(1.76-3.20)\end{array}$ & $\begin{array}{c}2.09 \\
(1.09-4.02)\end{array}$ & (5) \\
\hline Khan et $a l$ & 56,881 & $40-79$ & Cohort & $\begin{array}{c}\text { ICD } \\
\text { C22.0-9 }\end{array}$ & 5.8 & $\begin{array}{c}2.30 \\
(1.47-3.59)\end{array}$ & $\begin{array}{c}2.70 \\
(1.20-6.05)\end{array}$ & (6) \\
\hline Kuriki et al & 59,440 & $40-80$ & Cohort & ICD C22 & 4.2 & $\begin{array}{c}2.04 \\
(1.48-2.80)\end{array}$ & $\begin{array}{c}2.27 \\
(1.08-4.77)\end{array}$ & (7) \\
\hline Matsuo & 444 & $40-75$ & $\begin{array}{l}\text { Case- } \\
\text { control }\end{array}$ & $\mathrm{HCC}$ & 15.8 & $\begin{array}{c}2.52 \\
(1.27-5.02)\end{array}$ & $\begin{array}{c}4.20 \\
(0.81-21.81)\end{array}$ & (8) \\
\hline Present study & 74,869 & 40-104 & $\begin{array}{c}\text { Cross- } \\
\text { sectional }\end{array}$ & $\mathrm{HCC}$ & 9.3 & $\begin{array}{c}1.25 \\
(1.12-1.39)\end{array}$ & $\begin{array}{c}1.22 \\
(1.00-1.49)\end{array}$ & \\
\hline
\end{tabular}

CI, confidence interval; DM, diabetes mellitus; HCC, hepatocellular carcinoma; ICD, International Classification of Diseases; OR, odds ratio.

for the epidemiological evaluation of pathological alterations in DM cases.

Treatment with dipeptidyl peptidase (DPP)-IV inhibitors and glucagon-like peptide (GLP)-1 agonists has been suggested to induce the proliferation and dysplasia of ducts and hyperplasia of endocrine cells based on materials from the Network for Pancreatic Organ Donors with Diabetes (13). The pathological changes associated with incretin-based therapy were not reproduced by an intensive review of the same database (14). The increase in the risk of developing ICC by incretin-based therapy was not noted in a recent case-control study (15). In the present study, autopsy cases were registered between 2005 and 2009, which was before DPP-IV inhibitors had become available for the treatment of DM in Japan (16). Therefore, the ORs in the present study were not influenced by incretin-based therapy. DPP-IV inhibitors are currently used for the treatment of DM in Japan. It is plausible to examine the changes of diseases of the pancreas by the autopsy records after 2009 . 
In conclusion, in the present study, the association of DM with cancers was examined using the Annual of Pathological Autopsy Cases in Japan. Although the ORs estimated from autopsy records may warrant careful interpretation, the ORs of HCC were increased in both males and females with DM. To the best of our knowledge, this is the first study to demonstrate the association of HCC with DM using autopsy records.

\section{Acknowledgements}

Not applicable.

\section{Funding}

No funding was received.

\section{Availability of data and materials}

The data and material that support the findings of this study are available from the corresponding author upon reasonable request.

\section{Authors' contributions}

RW and ZN designed the study. The conversion of PDF files to Excel files and the reviewing of the data were performed by RW and SKi. Statistical analyses were performed by RW, SKu and KI. MK verified diabetic patients, and prepared the figure. All authors proofread and approved the final manuscript for publication.

\section{Ethics approval and consent to participate}

Not applicable.

\section{Patient consent for publication}

Not applicable.

\section{Competing interests}

The authors declare that they have no competing interests.

\section{References}

1. Giovannucci E, Harlan DM, Archer MC, Bergenstal RM, Gapstur SM, Habel LA, Pollak M, Regensteiner JG and Ye D: Diabetes and cancer: A consensus report. Diabetes Care 33: 1674-1685, 2010.
2. Kasuga M, Ueki K, Tajima N, Noda M, Ohashi K, Noto H, Goto A, Ogawa W, Sakai R, Tsugane S, et al: Report of the Japan diabetes society/Japanese cancer association joint committee on diabetes and cancer. Cancer Sci 104: 965-976, 2013.

3. El-Serag HB, Hampel H and Javadi F: The association between diabetes and hepatocellular carcinoma: A systematic review of epidemiologic evidence. Clin Gastroenterol Hepatol 4: 369-380, 2006.

4. Huxley R, Ansary-Moghaddam A, Berrington de González A and Woodward M: Type-II diabetes and pancreatic cancer: A meta-analysis of 36 studies. Br J Cancer 92: 2076-2083, 2005.

5. Inoue M, Iwasaki M, Otani T, Sasazuki S, Noda M and Tsugane S: Diabetes mellitus and the risk of cancer: Results from a large-scale population-based cohort study in Japan. Arch Intern Med 166: 1871-1877, 2006.

6. Khan M, Mori M, Fujino Y, Shibata A, Sakauchi F, Washio M and Tamakoshi A; Japan Collaborative Cohort Study G: Site-Specific cancer risk due to diabetes mellitus history: Evidence from the Japan collaborative cohort (JACC) study. Asian Pac J Cancer Prev 7: 253-259, 2006.

7. Kuriki K, Hirose $\mathrm{K}$ and Tajima K: Diabetes and cancer risk for all and specific sites among Japanese men and women. Eur J Cancer Prev 16: 83-89, 2007.

8. Matsuo M: Association between diabetes mellitus and hepatocellular carcinoma: Results of a hospital- and community-based case-control study. Kurume Med J 50: 91-98, 2003.

9. Japanese Society of Pathology: Annual report of pathological autopsy cases in Japan 2005-2009. Japanese Society of Pathology, Tokyo, 2006-2010.

10. Japanese Ministry of Health Labour and Welfare: Vital statistics of Japan final data. General Mortality, 2009. https://www.e-stat. go.jp/.

11. Jing W, Jin G, Zhou X, Zhou Y, Zhang Y, Shao C, Liu R and $\mathrm{Hu} X$ : Diabetes mellitus and increased risk of cholangiocarcinoma: A meta-analysis. Eur J Cancer Prev 21: 24-31, 2012.

12. Japanese Ministry of Health Labour and Welfare: The national health and nutrition survey in Japan, 2007. https://www.mhlw. go.jp/bunya/kenkou/eiyou09/01.html.

13. Butler AE, Campbell-Thompson M, Gurlo T, Dawson DW, Atkinson $\mathrm{M}$ and Butler PC: Marked expansion of exocrine and endocrine pancreas with incretin therapy in humans with increased exocrine pancreas dysplasia and the potential for glucagon-producing neuroendocrine tumors. Diabetes 62: 2595-2604, 2013.

14. Harja E, Lord J and Skyler JS: An analysis of characteristics of subjects examined for incretin effects on pancreatic pathology. Diabetes Technol Ther 15: 609-618, 2013.

15. Giorda CB, Picariello R, Tartaglino B, Nada E, Costa G and Gnavi R: Incretin-based therapy and risk of cholangiocarcinoma: A nested case-control study in a population of subjects with type 2 diabetes. Acta Diabetol 57: 401-408, 2020.

16. Goda $\mathrm{M}$ and Kadowaki T: Teneligliptin for the treatment of type 2 diabetes. Drugs Today (Barc) 49: 615-629, 2013.

(i) $\odot$ This work is licensed under a Creative Commons Attribution-NonCommercial-NoDerivatives 4.0 International (CC BY-NC-ND 4.0) License. 